\title{
A High Performance Skin Impedance Measurement Circuit for Biomedical Applications
}

\author{
K Hayatleh, S Zourob, R Nagulapalli,S Barker, N Yassine, P Georgiou*, F J Lidgey \\ Oxford Brookes University, Oxford, UK \\ *Imperial College, London, UK \\ khayatleh@brookes.ac.uk
}

\begin{abstract}
This paper describes a high-performance impedance measurement circuit for the application of skin impedance measurement in the early detection of skin cancer. A CMRR improvement technique has been adopted for OTAs to reduce the impact of high frequency common mode interference. A modified 3-OTA IA has been proposed to help with the impedance measurement. Such systems offer a quick, non-invasive and painless procedure, thus having considerable advantages over the currently used approach, which is based upon the testing of a biopsy sample. The sensor has been implemented in $65 \mathrm{~nm}$ CMOS technology and post layout simulations confirms the theoretical claims we made and sensor exhibits sensitivity. Circuit consumes $45 \mathrm{uW}$ from $1.5 \mathrm{~V}$ power supply. The circuit occupies $0.01954 \mathrm{~mm}^{2}$ silicon area.
\end{abstract}

Keywords- Skin cancer detection, non-invasive, CMRR; Differential pair, Input impedance, Instrumentation amplifier; Sensor, Sensitivity, Resistance.

\section{INTRODUCTION}

In recent days, skin cancer is seen as one of the most hazardous forms of cancer found in humans. Skin cancer is found in various types such as Melanoma, Basal and squamous cell Carcinoma among which Melanoma is the most unpredictable. The detection of melanoma cancer in early stage can be very helpful to cure. With increased flexibility and complex digital processing capability, electronics based cancer detection gained a lot of attention in the recent days [1]. In this paper, we proposed an impedance measurement circuit for use in the early detection of skin cancer based on Instrumentation amplifier (IA). Currently used techniques require the need for a biopsy, which is both invasive and painful. Additionally, the analysis of the sample takes some considerable time. Of course, all these procedures require the time of clinicians to perform them. The system proposed here will be based on accurate measurement of skin electrical resistance, meaning that the clinical diagnostic tests will be both non-invasive and painless, and can be performed by a non-clinician. A radiometric approach is taken, in that measurements are taken of both normal and abnormal skin of a patient, and the results compared, hence normalizing out any differences in skin types, thereby eliminating the misinterpretation of results due to this. Furthermore, the results of the tests will be easy to analyses, providing a rapid diagnosis for the patient, thus minimizing the distress for the patient caused by waiting for the results.

To date, several independent researchers have been and still are investigating the use of skin impedance measurements in various clinical situations. As a discipline, it has been of interest of a very long time [2] amongst the early research. Skin resistance has been of interest for some time for use as a clinical diagnostic tool for the detection of cancer. Weitzen [3] had some very interesting results with lung, breast, and prostate cancer patients: The work was based on the underlying principle that the skin resistance of specific dermal-visceral (DVZs) zones could reflect the pathological states of coinciding internal organs due to the pathological condition of internal organs inducing electrophysiological changes in these corresponding DVZs. Their studies have shown that the accuracy of predictions based on this technique was surprisingly high. Weitzen et al., [3] discussed the same technique in the detection of breast cancer using Electrical Impedance (Mammography) systems.

We have proposed a modification to the existing Instrumentation amplifier to be used as an impedance measurement circuit. There are three general approaches to implementing instrumentation amplifiers in this type of application [4-6]. The first approach involves the use of operational amplifiers with resistive feedback, but this gives often very low CMRR. The Second approach is the 3-OTA instrumentation amplifier which is the probably the most well-known approach. In this approach two OTAs are used to implement a fully differential buffer, which is followed by a single OTA configured as a difference amplifier, in this configuration CMRR is dominated by the first stage resistor mismatch and 2nd stage OTA CMRR. This approach has a significant problem with input and output common voltages (they must be very close). The use of Current Feedback Instrumentation amplifier solves common mode voltage problem but often comes with the gain trade-off. The last 
approach involves the use of switched capacitor techniques to overcome the common mode voltages but needs an onchip clock and large filter to suppress the ripple. In this paper the 3-OTAs architecture has been chosen because of its simplicity and since it doesn't need any clock. A traditional OTA with a simple bias often may offer limited usage in this application due to its CMRR limitation at high frequency, so a CMRR enhancement technique is exploited to improve the sensor sensitivity.

The rest of the paper has been organized as follows. Section-2 describes CMR bandwidth enhancement technique and rigorous qualitative and quantitative explanation has been given. Section-3 describes the very limited properties of skin cell and electrical model of the cell. Section-4 describes the proposed technique.

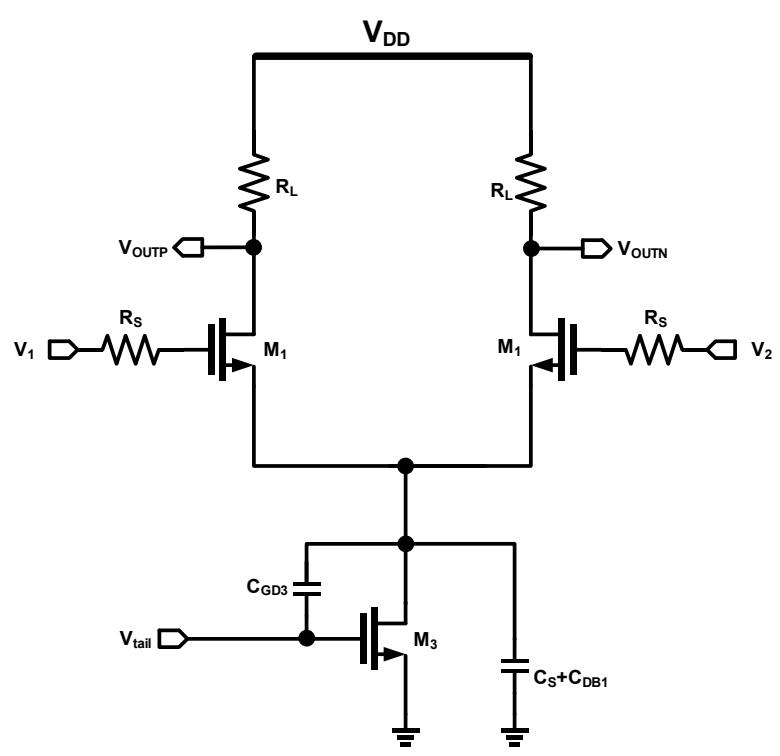

Fig. 1. Basic differential pair

\section{BANDWIDTH ENHANCEMENT TECHNIQUE}

Fig. 1 Shows a generic source coupled differential pair with a resistive load. Often this kind of amplifiers needs to amplify weak differential signals that are often accompanied by strong common-mode (CM) signals. For example, differential signals ranging from $10 \mathrm{~V}$ to $100 \mathrm{mV}$ with a $\mathrm{CM}$ voltage ranging from $1.9 \mathrm{~V}$ to $5 \mathrm{~V}$. Amplifying such weak signals require an amplifier with an offset below $10 \mathrm{uV}$ and a CMRR more than $100 \mathrm{~dB}$, which is quite challenging. Additionally, the bandwidth needs to be wide enough to minimize any harmonic distortion to acceptable levels for the application. This is very important in biomedical applications where a misrepresentation of the signals involved could lead to misdiagnosis [7].
Amplifier inputs $V_{1}$ and $V_{2}$ can be expressed as differential and common mode components as given by the following equations.

$$
\mathrm{V}_{1}=\mathrm{V}_{\mathrm{c}}+\frac{\mathrm{V}_{\mathrm{d}}}{2} \text { and } \mathrm{V}_{2}=\mathrm{V}_{\mathrm{c}}-\frac{\mathrm{V}_{\mathrm{d}}}{2}
$$

Where $V_{c}$ is common mode input and $V_{d}$ is differential mode input. Amplifier output can be expressed as

$$
V_{0}=A_{d} V_{d}+A_{C} V_{C}
$$

Where $A_{c}$ is the common mode gain and $A_{d}$ is the differential mode gain. CMRR can be defined as

$$
C M R R=\frac{A_{d}}{A_{c}}
$$

Where $A_{d}$ and $A_{c}$ are frequency dependent and hence CMRR also frequency dependent. By applying small signal model to the Fig. 1 amplifier Differential mode gain can be derived as the follows

$$
A_{d}=\frac{G_{m} R_{L}^{1}}{1+\frac{S}{\omega_{p d}}}
$$

Where $\mathrm{G}_{\mathrm{m}}$ is differential pair small signal trans-conductance and $R_{L}^{1}$ is the parallel combination of $\mathrm{R}_{\mathrm{L}}$ and $\mathrm{R}_{0}$ (transistor $\mathrm{o} / \mathrm{p}$ resistance). $\omega_{\mathrm{pd}}$ gives differential gain dominate pole given by

$$
\omega_{\mathrm{pd}}=\frac{1}{\mathrm{R}_{\mathrm{S}} \mathrm{C}_{\mathrm{gs}}+\mathrm{R}_{\mathrm{S}} \mathrm{C}_{\mathrm{gd}}\left(1+\mathrm{G}_{\mathrm{m}} R_{L}^{1}\right)+R_{L}^{1}\left(\mathrm{C}_{\mathrm{L}}+\mathrm{C}_{\mathrm{gd}}\right)}
$$

Where $\mathrm{C}_{\mathrm{L}}$ is the load capacitance at output of the differential pair, and $\mathrm{C}_{\mathrm{gd}}, \mathrm{C}_{\mathrm{gs}}$ are the transistor gate to source and gate to drain capacitances.

Common-mode gain can be defined as

$$
\mathrm{A}_{\mathrm{C}}=\frac{-R_{L}^{1}}{2 \mathrm{R}_{\mathrm{T}}} \frac{\left(1+\mathrm{SR}_{\mathrm{L}} \mathrm{C}_{\mathrm{T}}\right)}{1+\mathrm{S} R_{\mathrm{L}}^{1} \mathrm{C}_{\mathrm{T}}}
$$

From the equation (6) $\mathrm{A}_{\mathrm{c}}$ exhibits a pole and a zero. Where $\mathrm{R}_{\mathrm{T}}$ is the impedance looking into the tail transistor and $\mathrm{C}_{\mathrm{T}}$ is the capacitance looking into the tail transistor drain. $\mathrm{C}_{\mathrm{T}}$ consists of three capacitances, $\mathrm{C}_{\mathrm{S}}, \mathrm{C}_{\mathrm{db} 3}, \mathrm{C}_{\mathrm{gb} 3}$

$$
\mathrm{C}_{\mathrm{T}}=\mathrm{C}_{\mathrm{s}}+\mathrm{C}_{\mathrm{db} 3}+\mathrm{C}_{\mathrm{gb} 3}
$$

Where $C_{S}$ is the stray capacitance at the common source node of the differential pair. Clearly common-mode gain zero frequency is very less compared to pole frequency because $\mathrm{R}_{\mathrm{T}}^{1} \gg \mathrm{R}_{\mathrm{L}}$. From equation (3), Zeros of common mode gain will be the pole of CMRR, so from the above description, the $3 \mathrm{~dB}$ bandwidth of the CMRR is:

$$
\mathrm{CMRR}=\frac{1}{\mathrm{R}_{\mathrm{T}} \mathrm{C}_{\mathrm{T}}}
$$

Gain bandwidth product of CMRR is: 


$$
G B W=\frac{\mathrm{G}_{\mathrm{m}}}{\mathrm{C}_{\mathrm{T}}}
$$

So, for a specified and practically achievable CMRR, the only way to improve the bandwidth is either maximize the $G_{m}$ or decrease $C_{T}$ and maximizing $G_{m}$ only possible by increasing current which is costly in terms of the power consumption. Generally high CMRR is required to minimize the common mode noise at the output. Common mode noise sources like $50 \mathrm{~Hz}$ power supply noise, 10$20 \mathrm{KHz}$ switching noise originated from switching converters could interfere with the weak biomedical input signals, so a high CMRR value needs to be maintained up to these high frequencies to minimise power supply noise. Hence, the signal to noise ratio (SNR) of a bio-medical transceiver will be improved by increasing the CMRR bandwidth. A technique to decrease tail node capacitance by neutralization has been presented here.

Fig. 2 shows proposed solution [8] to enhance the CMRR bandwidth. Additional externally biased transistor $\mathrm{M}_{4}$ is incorporated into the circuit to compensate gate to drain capacitance of transistor $\mathrm{M}_{3}$ and its drain is connected to $\mathrm{M}_{3}$ Source. A change in the drain voltage of $\mathrm{M}_{3}$ gives rise to a change $\mathrm{I}_{\mathrm{J}}$ in the gate-drain capacitance of $\mathrm{M}_{3}$. A part, $\mathrm{I}_{\mathrm{K}}$, of $\mathrm{I}_{\mathrm{J}}$ is returned to the source circuit $\mathrm{M}_{3}$. Hence at the drain of $\mathrm{M}_{3}, \mathrm{C}_{\mathrm{gd} 3}$ appears to be a substantially.

$$
C_{d g 3}^{\prime} \sim C_{g d 3}\left[1-\frac{I_{k}}{I_{J}}\right]
$$

Total capacitance at the drain of $\mathrm{M}_{3}$, which was designated $\mathrm{C}_{\mathrm{T}}$ in Fig. 1, now becomes $\mathrm{C}_{\mathrm{T} 1}$

$$
C_{T}^{\prime}=C_{S}+C_{d b 3}+C_{g b 3}+\left[1-\frac{I_{k}}{I_{J}}\right] C_{g d 3}
$$

From equation (11) it is very clear that CMRR bandwidth has been extended. Like any positive feedback, this technique also can potentially make circuit unstable by making the capacitance negative, so care must be taken to make sure $C_{S}+C_{d b 3}+C_{g b 3}$ is always greater than the $\left[1-\frac{I_{k}}{I_{J}}\right] C_{g d 3}$. In this design, we swept $\mathrm{I}_{\mathrm{K}}$ and simulated the CMRR. Up to certain extent the bandwidth will increase because the capacitance will decrease, and beyond this $\mathrm{I}_{\mathrm{K}}$ value the circuit will latch due to the +ve feedback. It is therefore important that the $I_{K}$ value doesn't latch to the rails by avoiding -ve capacitance. Fig. 3 shows the CMRR plot for the conventional circuit (fixed bias current source differential pair) and the proposed bias. Fig. 3 shows that with the conventional bias CMRR, the $3-\mathrm{dB}$ bandwidth is $11.3 \mathrm{KHz}$, and with the proposed biasing technique it has been extended to $27.24 \mathrm{KHz}$, which is more than a $100 \%$ improvement.

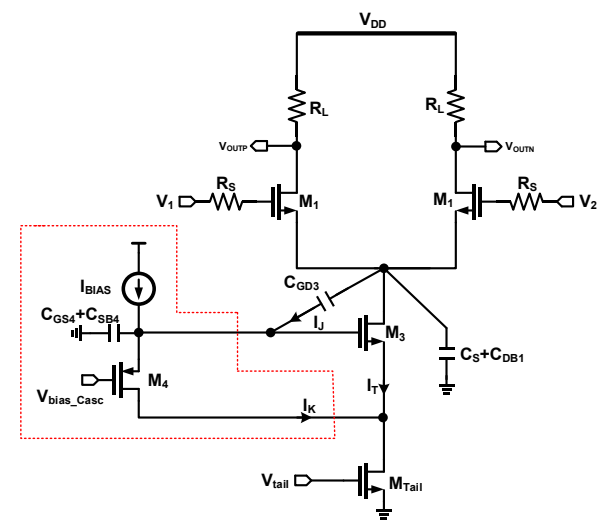

Fig. 2. CMRR enhancement technique

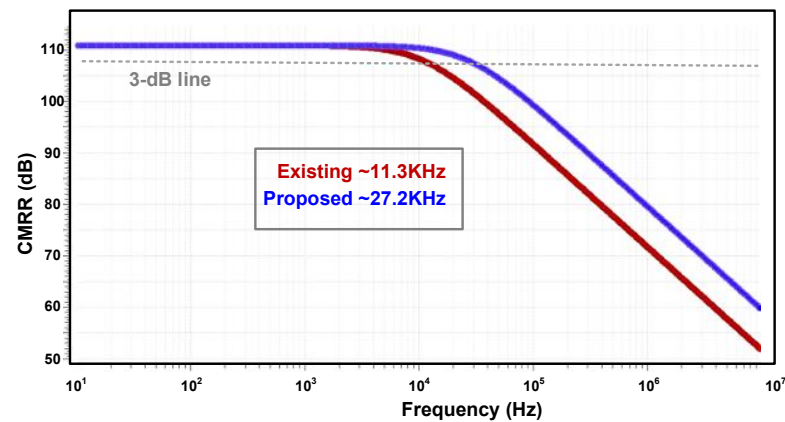

Fig. 3. CMRR frequency response.

CMRR is generally very sensitive to the process, voltage, and temperature (PVT) variations, because from the equation (6) the numerator depends on the load resistance $\mathrm{R}_{\mathrm{L}}$ which is typically made of poly-silicon and typically varies by $10 \%$. The denominator depends on the $\mathrm{M}_{3}$ small signal output resistance $R_{0}$, which varies more than $25 \%$ across PVT. Unfortunately, both $\mathrm{R}_{\mathrm{L}}$, and $\mathrm{R}_{\mathrm{o}}$ vary due to different physical effects, hence there will be a maximum $35 \%$ PVT variation in CMRR. Fig. 4 shows the simulated low frequency CMRR across PVT corners, and displays $\sim 32 \%$ variation.

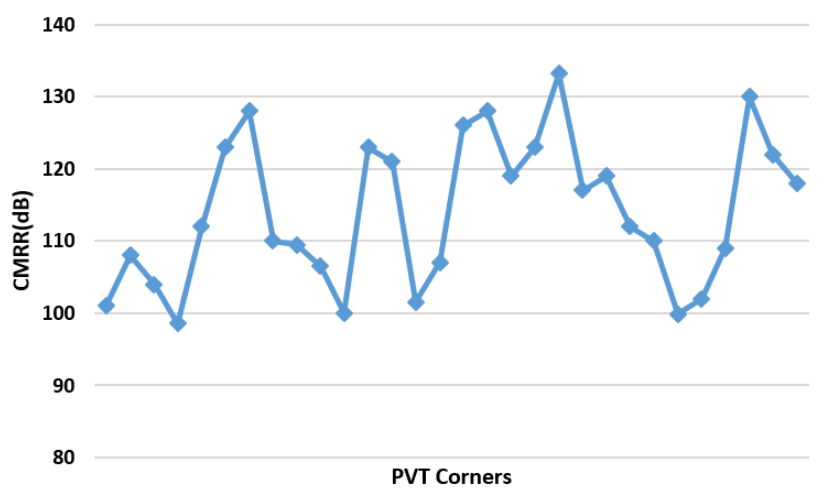

Fig. 4. CMRR Variation across PVT corners. 


\section{SKIN TISSUE MODEL}

Living cell membranes can be considered as electrochemical in nature, thus allowing some ions to pass through, while others are blocked. This can be considered analogous to a leaky capacitor. The cell itself, along with its environment largely consists of electrolytes which have resistive characteristics.

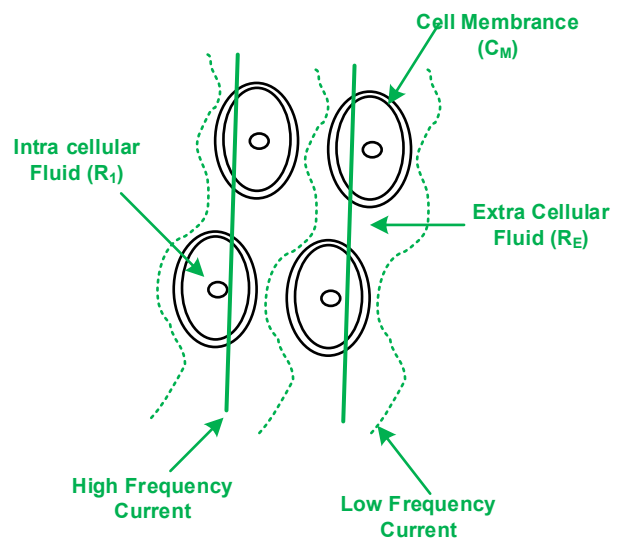

Fig. 5. Current flow in Tissue.

Fig. 5 shows the current flow in the tissue, when electricity is passed through the body, two types of impedances that are capacitive (reactive) $\mathrm{C}$ and resistive $\mathrm{R}$ are offered by the body, where capacitance arises due to the cellular membrane and resistance arises due to the body water (could be intracellular or extracellular). Cell membrane consists of a layer of nonconductive lipid material sandwiched between two layers of conductive protein molecules. The high reactive value indicates good health and cell membrane integrity. Cell membrane structure makes them behave as a capacitor when the alternating current is applied to it. Hence impedance of tissue varies with frequency. At high frequency, current can flow through both intra and extra cellular water which means that it can penetrate the cellular membrane while at low-frequency current cannot penetrate cellular membrane so it flows only through extracellular fluid. Thus, at low frequency, impedance is resistive in nature and at high frequency, it has a resistive as well as a reactive component [9]. As the cells have a capacitive element, DC, and low-frequency AC must pass around the cell rather than through it. Conversely, at higher frequencies current can pass through the cell itself. Ultimately, this means differing types of tissue show a different behavior to different frequency bands. This means that a model of tissue can be developed using resistive and capacitive elements [10] [11] as shown below in Fig. 6. Resistances $R_{1}, R_{E}$ represents the Intra and extra Cellular fluid effects respectively and $C_{M}$ represents the membrane capacitance. The frequency response of the impedance can be expressed as (12) and shows its low-frequency value is $R_{1}+R_{E}$ and high-frequency value goes down to $R_{1}$ due to the capacitor [12]. It has a pole and zero in the frequency response as given in (13). Fig. 7 shows the frequency response of the tissue impedance.

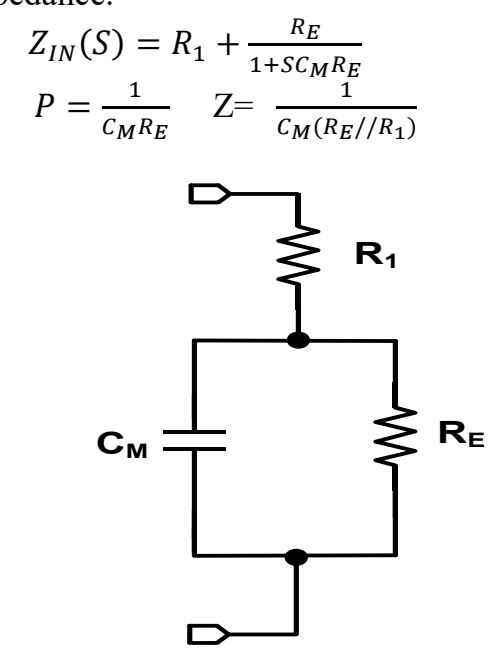

Fig. 6. Tissue model.

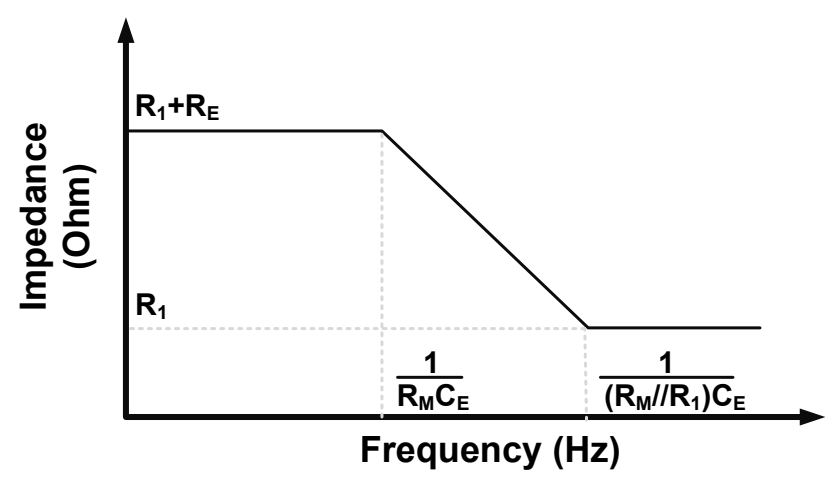

Fig. 7. Tissue Frequency Responce.

Typical values for the $\mathrm{R}_{\mathrm{E}}$ and $\mathrm{C}_{\mathrm{M}}$ are $8 \mathrm{k}$ and $1500 \mathrm{pF}$. The Frequency response of the impedance corresponding to any Skin tissue is low pass nature with approximately $13.26 \mathrm{kHz} 3 \mathrm{~dB}$-bandwidth due to shunt capacitance $\mathrm{C}_{\mathrm{M}}$. Any change in the Tissue impedance nature at a given frequency is the clear symptom of cancer or some decease related to the skin.

In the literature, there hasn't been any research pertaining towards skin cancer. There have been several ways to measure the impedance, often people tend to use Trans-impedance amplifier (TIA) due to its simple nature, requires only a high gain amplifier and biasing circuit, but unfortunately it consume smore power and noisy. [4] proposed an impedance measurement system, but involves lot of Digital Signal processing. In this paper, we explored a modified Instrumentations-Amplifier (IA) to measure the impedance and proposed a way to quantify the level of skin cancer. 


\section{PROPOSED IC DESIGN}

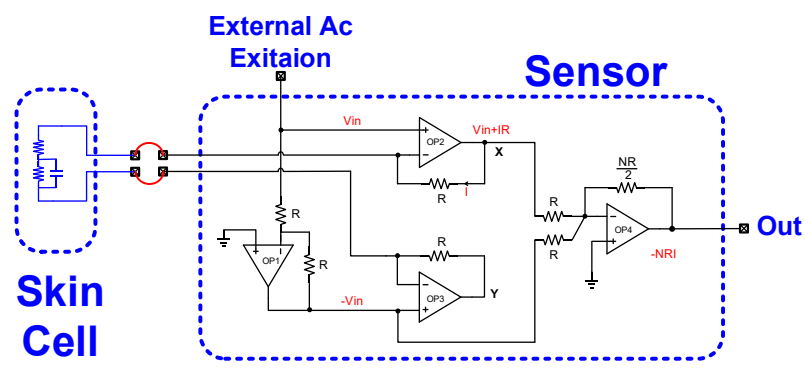

Fig. 8. Proposed IA-based circuit.

Fig. 8 depicts our proposed impedance measurement circuit derived from 3-OTA instrumentation amplifier. Generally, instrumentation amplifier requires two voltages as inputs and will be converted into a current. Here an accurate external low-frequency signal is used to excite the circuit through non-inverting input of the op-amp OP2 and through Inverting amplifier OP1 complimentary input signal -vin has been generated. The voltage across the sensor is $2 \mathrm{~V}_{\text {in }}$, provided the OTA $\left(\mathrm{OP}_{1}\right)$ offset is very low, and the feedback resistors around $\mathrm{OP}_{1}$ match very well. Any offset or mismatch in the components will result in errors at the output of the inverting amplifier will could lead to a clinical misdiagnosis. To minimize the mismatch induced offset, $\mathrm{OP}_{1}$ has been designed with larger physical size components. Fig. 9 shows the simulated gain of the inverting amplifier formed by $\mathrm{OP}_{1}$ with the component mismatch (Monte-Carlo Simulation). The histogram shows unity gain with $1.9 \%$ of standard deviation, which roughly indicates that the output will have $1.9 \%$ error. A voltage to current conversion will occur and current will flow through OP2 and OP3 feedback resistors. Node x (OP2 output) voltage is depending on the input voltage and current through the sensor. Since we are interested only sensor current ideally we should subtract input signal voltage from node $\mathrm{x}$, so we used summer as a final stage. All node voltages and branch currents have been highlighted in in Fig. 9. Common mode rejection of the final summer amplifier impacts the sensitivity of the sensor, so the current CMRR enhancement technique would help a lot here. To measure the impedance, change due to cancer attack, few parameters of this circuit can be measured as detection parameter. The current through the skin cell give very good indication because voltage across the skin cell is $2 \mathrm{~V}_{\mathrm{IN}}$ hence current depends on the impedance but this current must be much lesser than $350 \mathrm{uA}$ due to safety limits, otherwise cell properties will change. The voltage output voltage of the sensor also gives reasonable estimation of the impedance, hence cancer level. The sensitivity of the of the circuit can be defined as a change in the output voltage per unit sensor capacitance for a given input signal.

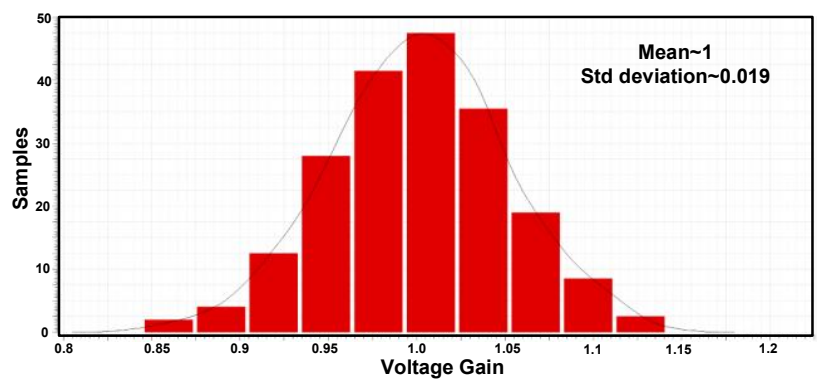

Fig. 9. Inverting Amplifier Gain Histogram.

A two-stage folded cascode OTA [13] architecture show in Fig. 10 has been chosen for its input and output common voltage compatibility and High gain. As explained in the previous sections, CMRR bandwidth enhancement tech also been adopted, so that this sensor can accepted much higher frequency of input signal due to the proposed bias technique without having any power or performance overhead. To achieve closed loop settling error less than $0.1 \%$, the loopgain of the any negative feedback loop in the circuit should be greater than $60 \mathrm{~dB}$ across all PVT (process, voltage, Temperature) corners. To as to maintain less gain variation and minimal input referred offset, self-bias technique has been adopted [13]. Fig. 11 shows the loop gain of the amplifier with and without self-bias technique, shows significant improvement. Since it is a two stage OTA, need to compensate for the well behaved transient response otherwise it may oscillate or even compromises the sensitivity, so miller compensation has been used by placing a capacitor $\left(C_{C}\right)$ across the second stage. From the stability point of view summing amplifier (consists of OP4) is difficult one because of the higher feedback ratio. Fig. 12 shows the frequency response of the closed loop response, shows $55^{0}$ phase margin. Each unit resistor used in the design is $1 \mathrm{k} \Omega$ and Value of $\mathrm{N}$ (the resistor ratio shown in Fig. 5) is 60 . The power supply rejection of the op-amp is around $-70 \mathrm{~dB}$ in the frequency of interest such that it will not impact sensor accuracy.

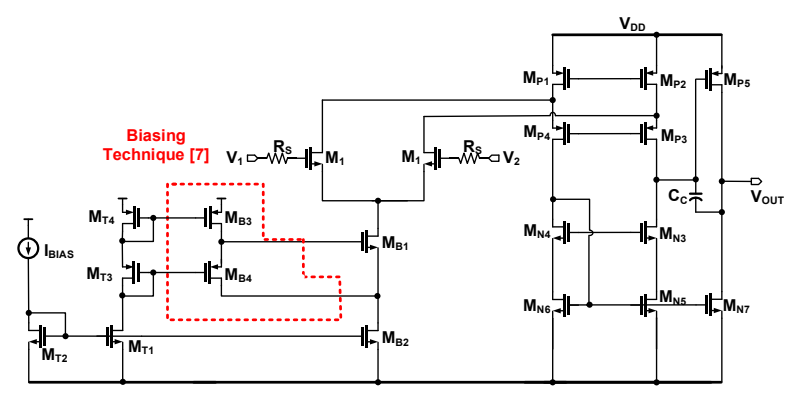

Fig. 10. OTA architecture used for Sensor design. 


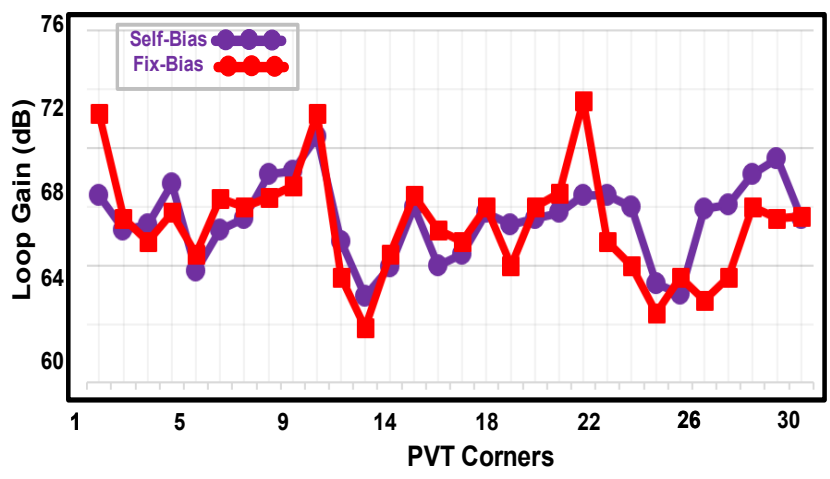

Fig. 11. Low frequency Loop-Gain across PVT corners

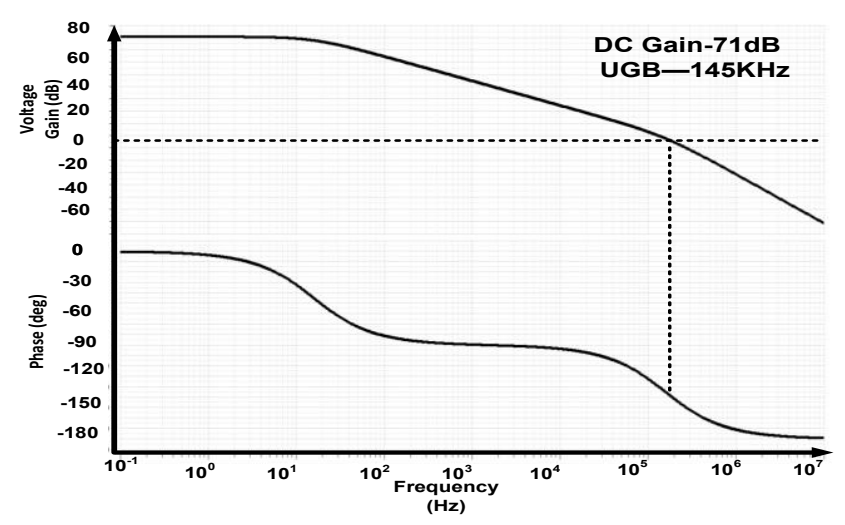

Fig. 12. Frequency response of the closed loop

\section{SENSOR RESULTS}

The impedance sensor instrumentation has been implemented in $65 \mathrm{~nm} 1 \mathrm{P} 8 \mathrm{M}$ CMOS technology and post layout simulations have been performed. Tissue probes connections are explicitly placed below the amplifiers so that it can be integrated without having difficulty in assigning power connections. Post-layout simulations have been performed on the final layout. We applied a $10 \mathrm{mV}$, $10 \mathrm{KHz}$ sinusoidal excitation for the sensor and Fig. 13 shows the current through skin cell and voltage at the output of the circuit while sweeping the cell model capacitance from $1500 \mathrm{pF}$ to $3500 \mathrm{pF}$. As explained in section-IV, for a given input signal amplitude, the sensor output voltage linearly varies with skin tissue capacitance. Over the range of tissue capacitance output voltage has been changed by $47 \mathrm{mV}$ hence this sensor has a sensitivity of $23.5 \mathrm{uV} / \mathrm{pF}$. The sensitivity we have expressed is directly related to the capacitance. Higher the sensor output, severe the skin cancer attach.

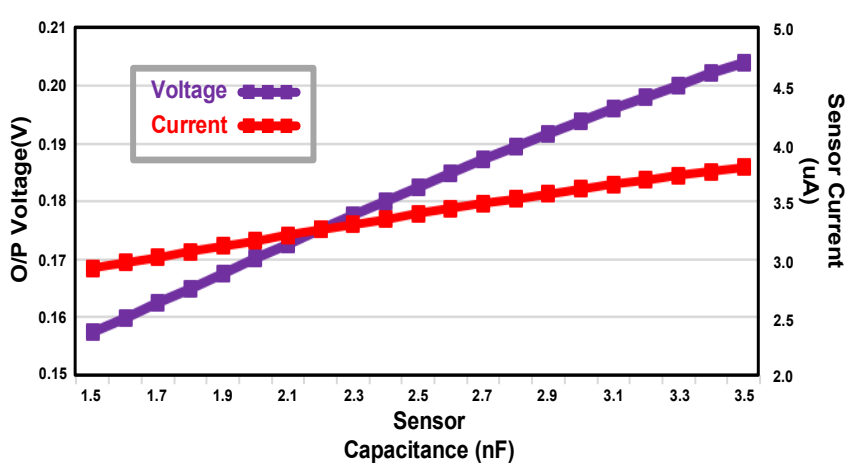

Fig. 13. Sensor output voltage vs input capacitance.

Fig. 14 shows the frequency response of the sensor, which depicts sensor output voltage versus frequency of the applied signal for a given capacitance. At all frequencies, the output voltage increases with capacitance. Results in Fig. 13 and Fig. 14 are agreeing with each other's apart from the fact that one is in the time domain and another is in the frequency domain. Fig. 15 shows the layout of the proposed sensor, it occupies $0.01914 \mathrm{~mm}^{2}$ active silicon area, most of the area has been occupied by the resistors and compensation capacitor. Used typical layout techniques like common centroid and inter-digitization of the current mirrors to minimize the linear gradient proposes variations. Layout.

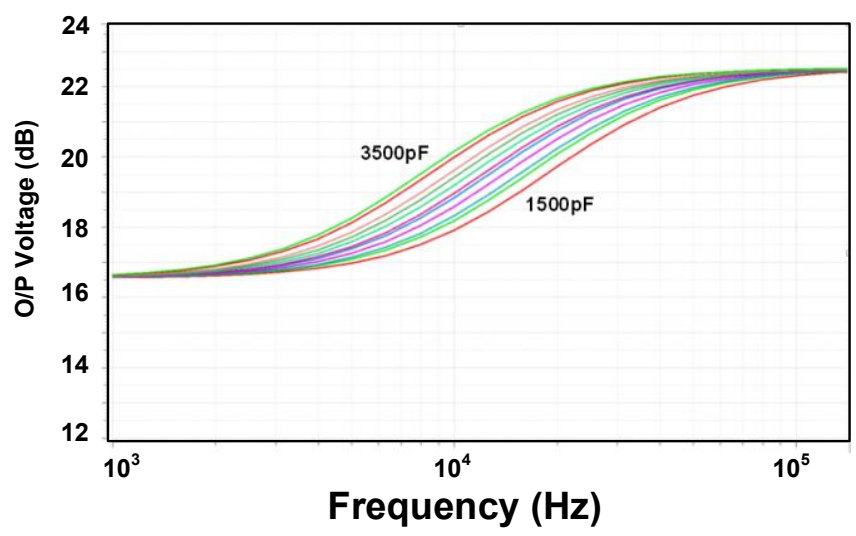

Fig. 14. Frequency response of the sensor. 


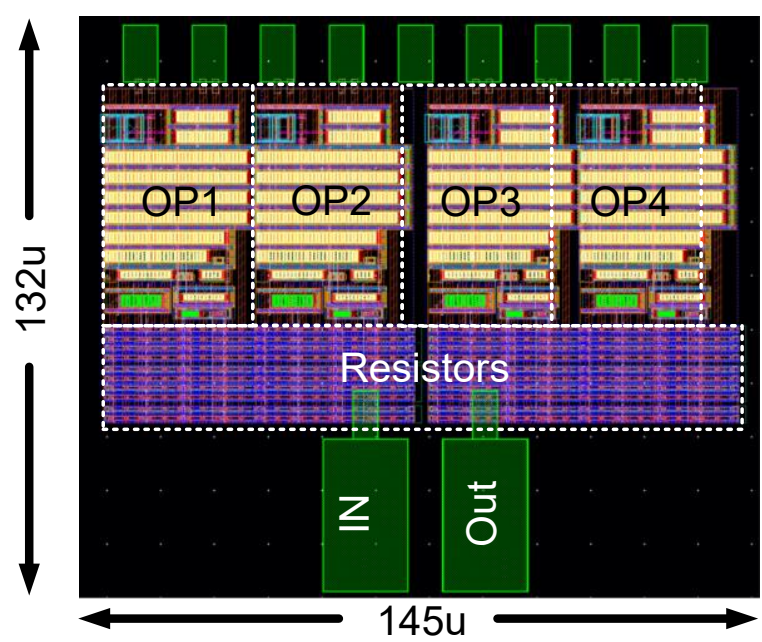

Fig. 15. The layout of the sensor

\section{CONCLUSION}

We have presented a skin impedance measuring circuit that can be used to evaluate and compare the electrical properties of normal and abnormal Epidermis, hence providing the possibility of the early detection of skin cancer. According to the authors knowledge this is the first skin sensor CMOS technology IC in the literature. A 2.5 times improvement in CMRR bandwidth has also been demonstrated for the differential amplifier. A sensitivity of $23.5 \mathrm{uV} / \mathrm{pF}$ demonstrated. As a future work, need to find a way to convert output voltage into digital domain, probably by adding ADC, so that it can be very easily integrated to a DSP to process the and store the results. The circuit occupies an area of $132 \times 145 \mathrm{um}^{2}$

\section{REFERENCES}

[1] Nagulapalli, R., Hayatleh, K., Barker, S. et al. Analog Integr Circ Sig Process (2017) 92: 437. https://doi.org/10.1007/s10470-017-1008-1

[2] Aveling, F., and McDowall, R. J. S. (1925). The effect of the circulation on the electric resistance of the skin. J. Physiol. (Lond.), 60, 316-321.

[3] Weitzen R, Epstein N, Shoenfeld Y, Zimlichman E. Diagnosing diseases by measurement of electrical skin impedance: a novel technique. Ann N Y Acad Sci. 2007;1109(1):185-192. doi:10.1196/annals.1398.022.

[4] A. Bakker, K. Thiele, J. H. Huijsing, "A CMOS nested-chopper instrumentation amplifier with 100-nV offset", IFEE J. Solid-State Circuits, vol. 35, no. 12, pp. 1877-1883, Dec. 2000.

[5] R. Wu, K. A. A. Makinwa, J. H. Huijsing, " A chopper currentfeedback instrumentation amplifier with a $1 \mathrm{mHz}|\$ 1 / \mathrm{f}| \$$ noise corner and an AC-coupled ripple reduction loop ", IEEE J. Solid-State Circuits, vol. 44, no. 12, pp. 3232-3243, Dec. 2009.

[6] M. Degrauwe, "A micropower CMOS-instrumentation amplifier", IEEE J. Solid-State Circuits, vol. sc-20, no. 3, pp. 805807, Jun. 1985.

[7] C. Hwang-Cherng and W. Jia-Yu, "High CMRR instrumentation amplifier for biomedical applications," in Signal Processing and Its Applications, 2007. ISSPA 2007. 9th International Symposium on, 2007, pp. 1-4.
[8] M. Ben-Esmael, B. L Hart, K. hayatleh, and F. J. Lidgey,' CMRR Bandwidth Extension Techniques for CMOS Differential Amplifiers, in the International Journal of Electronics and Communications, April 2014.

[9] K. R. Foster and H. P. Schwan, "Dielectric-Properties of Tissues and Biological-Materials - a Critical-Review," Critical Reviews in Biomedical Engineering, vol. 17, pp. 25-104, 1989.

[10] Hong, H. (2010) Novel CMOS Integrated Current Drivers for Wideband Bioimpedance Measurements, PhD Thesis, University College, London

[11] K. S. Cole, Electric impedance of suspension of spheres," TheJournal of General Physiology, vol. 12, no. 1, pp. 29-36, September 1928

[12] U. G. Kyle, I. Bosaeus, A. D. D. Lorenzo, P. Deurenberg, M. Elia, J. M. Gómez, B. L. Heitmann, L. Kent-Smith, J.-C. Melchior, M. Pirlich, H. Scharfetter, A. M. W. J. Schols and C. Pichard, "Bioelectrical impedance analysis - part I: review of principles and methods," Clinical Nutrition, vol. 23, pp. 1226-1243, 2004.

[13] R. Nagulapalli, K. Hayatleh, S. Barker, S. Zourob, N. Yassine, Sriadibhatla Sridevi, "A Microwatt Low Voltage Bandgap Reference for Bio-medical Applications", Recent Advances in Electronics and Communication Technology (ICRAECT) 2017 International Conference on, pp. 61-65, 2017. 